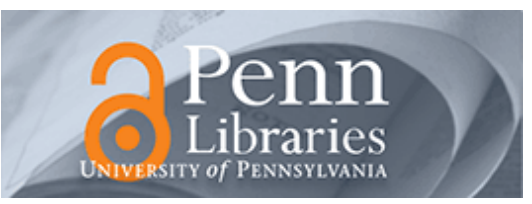

University of Pennsylvania

ScholarlyCommons

August 1998

\title{
Atomic Force Microscopy Study of an Ideally Hard Contact: The Diamond(111)/Tungsten Carbide Interface
}

\author{
M. Enachescu \\ University of Bucharest \\ R.J. A. van den Oetelaar \\ Eindhoven University of Technology \\ Robert W. Carpick \\ University of Pennsylvania, carpick@seas.upenn.edu \\ D. F. Ogletree \\ University of California \\ C. F. J. Flipse
}

Follow this and additional works at: https://repository.upenn.edu/meam_papers

See next page for additional authors

\section{Recommended Citation}

Enachescu, M.; van den Oetelaar, R.J. A.; Carpick, Robert W.; Ogletree, D. F.; Flipse, C. F. J.; and Salmeron, Miguel, "Atomic Force Microscopy Study of an Ideally Hard Contact: The Diamond(111)/Tungsten Carbide Interface" (1998). Departmental Papers (MEAM). 94.

https://repository.upenn.edu/meam_papers/94

Copyright (1998) American Institute of Physics. This article may be downloaded for personal use only. Any other use requires prior permission of the author and the American Institute of Physics. Reprinted in Physical Review Letters, Volume 81, Issue 9, August 1998, pages 1877-1879.

Publisher URL: http://dx.doi.org/10.1103/PhysRevLett.81.1877

NOTE: At the time of publication, author Robert W. Carpick was affiliated with the University of Wisconsin. Currently (June 2007), he is a faculty member in the Department of Mechanical Engineering and Applied Mechanics at the University of Pennsylvania.

This paper is posted at ScholarlyCommons. https://repository.upenn.edu/meam_papers/94

For more information, please contact repository@pobox.upenn.edu. 


\title{
Atomic Force Microscopy Study of an Ideally Hard Contact: The Diamond(111)/Tungsten Carbide Interface
}

\author{
Abstract \\ A comprehensive nanotribological study of a hydrogen-terminated diamond(111)/tungsten carbide \\ interface has been performed using ultrahigh vacuum atomic force microscopy. Both contact \\ conductance, which is proportional to contact area, and friction have been measured as a function of \\ applied load. We demonstrate for the first time that the load dependence of the contact area in UHV for \\ this extremely hard single asperity contact is described by the Derjaguin-Müller-Toporov continuum \\ mechanics model. Furthermore, the frictional force is found to be directly proportional to the contact \\ area.
}

\section{Comments}

Copyright (1998) American Institute of Physics. This article may be downloaded for personal use only. Any other use requires prior permission of the author and the American Institute of Physics. Reprinted in Physical Review Letters, Volume 81, Issue 9, August 1998, pages 1877-1879.

Publisher URL: http://dx.doi.org/10.1103/PhysRevLett.81.1877

NOTE: At the time of publication, author Robert W. Carpick was affiliated with the University of Wisconsin. Currently (June 2007), he is a faculty member in the Department of Mechanical Engineering and Applied Mechanics at the University of Pennsylvania.

\section{Author(s)}

M. Enachescu, R.J. A. van den Oetelaar, Robert W. Carpick, D. F. Ogletree, C. F. J. Flipse, and Miguel Salmeron 


\title{
Atomic Force Microscopy Study of an Ideally Hard Contact: The Diamond(111)/ Tungsten Carbide Interface
}

\author{
M. Enachescu, ${ }^{*}$ R. J. A. van den Oetelaar, ${ }^{\dagger}$ R. W. Carpick, ${ }^{\dagger}$ D. F. Ogletree, C. F. J. Flipse, ${ }^{\dagger}$ and M. Salmeron ${ }^{\S}$ \\ Materials Sciences Division, Lawrence Berkeley National Laboratory, University of California, Berkeley, California 94720
}

(Received 17 February 1998)

\begin{abstract}
A comprehensive nanotribological study of a hydrogen-terminated diamond(111)/tungsten carbide interface has been performed using ultrahigh vacuum atomic force microscopy. Both contact conductance, which is proportional to contact area, and friction have been measured as a function of applied load. We demonstrate for the first time that the load dependence of the contact area in UHV for this extremely hard single asperity contact is described by the Derjaguin-Müller-Toporov continuum mechanics model. Furthermore, the frictional force is found to be directly proportional to the contact area. [S0031-9007(98)06960-9]
\end{abstract}

PACS numbers: 62.20.Qp, 73.40.Cg

The atomic force microscope (AFM) has emerged as an important tool in nanotribological studies since the first observation of atomic-scale friction [1]. Many AFM friction experiments are carried out under atmospheric or inert gas conditions, where contamination [2] and water meniscus formation [3] significantly affect the results. With diamond, for example, sliding can catalyze a phase change in moderately evacuated chambers due to residual oxygen and water vapor [4]. Ultrahigh vacuum (UHV) experiments are therefore necessary to provide a reliable and fundamental insight into the relation between friction and contact area.

Recent UHV-AFM experiments on layered materials indicate that friction is proportional to the contact area for a nanometer-sized contact. Carpick et al. [5] found that friction was proportional to the contact area as described by the Johnson-Kendall-Roberts (JKR) model [6] for mica samples. Experiments by Lantz et al. [7] on $\mathrm{NbSe}_{2}$ and graphite resulted in a relation between friction and contact area described by the Maugis-Dugdale (MD) model [8]. These continuum mechanics models can, in principle, only be applied for isotropic materials, and corrections may be significant for layered materials [9]. No observations of the Derjaguin-Müller-Toporov (DMT) model [10] in UHV have been reported yet. A DMT dependence is predicted to occur for the case of stiff materials with low adhesion, such as the system under study. As will be discussed below, an ambiguity between the JKR and MD models makes application of the DMT model more straightforward.

In this Letter, we present a comprehensive nanotribological study of a hydrogen-terminated diamond(111)/ tungsten carbide single asperity contact using UHV-AFM. Diamond and tungsten carbide are two of the hardest, stiffest materials known, while the adhesive forces at the interface are small due to the hydrogen passivation of the diamond surface and to the fact that carbides are generally quite inert. These properties make the system under study an excellent candidate to exhibit a DMT-like behavior for contact area versus load. Furthermore, the mate- rials possess cubic symmetry, making the application of continuum mechanics models more justifiable compared to layered materials. Since the diamond sample is boron doped and tungsten carbide is conductive, local contact conductance measurements are performed as a function of applied load to obtain independent information about the contact area, in addition to the friction measurements.

Diamond and diamondlike films are important coating materials used in a wide variety of tools, hard disks, micromachines, and aerospace applications [11,12]. Similarly, tungsten carbide plays an important role in several types of hard coatings [13].

Only a few UHV-AFM studies on diamond singlecrystal surfaces have been performed so far. Germann et al. [14] and van den Oetelaar et al. [15] observed atomic-scale stick-slip features on the hydrogenterminated diamond(100) surface and diamond(111) surface, respectively. Furthermore, van den Oetelaar et al. [15] observed an enormous increase in friction after the removal of hydrogen. Harrison and co-workers [16] have carried out extensive molecular dynamics simulations of friction between diamond(111) surfaces, making diamond(111) an excellent model system.

The area $A$ of the interface of an asperity contact is an important parameter, since we may expect that the frictional force $F_{f}$ is proportional to it [17]: $F_{f}=\tau A$, where $\tau$ is the interfacial shear strength. The interfacial shear strength is a fundamental property, which may be constant or pressure dependent [18]. Therefore the pressure dependence of $\tau$ can be obtained by measuring $F_{f}$ and knowing the relation between $A$ and the applied load $L$. In most cases, this relation is calculated from elastic continuum mechanics models which describe the contact area between a sphere (tip) and a plane (sample) [9]. However, the load dependence of the contact area not only depends on the exact tip geometry but also on the strength of the adhesive forces compared to the elastic deformations [8,19-21].

The JKR and DMT models apply for two extreme cases: for compliant materials with large, short-range attractive 
forces and for stiff materials with small, long-range attractive forces, respectively. The empirical nondimensional parameter $\mu=\left(R \gamma^{2} / E^{* 2} z_{0}^{3}\right)^{1 / 3}$ can be used to determine which of the two models is most appropriate [20]. In this expression, $R$ is the sphere radius, $\gamma$ is the work per unit area required to separate tip and surface from contact to infinity, and $E^{*}$ is a combined elastic modulus, given by the equation $E^{*}=\left[\left(1-\nu_{1}^{2}\right) / E_{1}+\left(1-\nu_{2}^{2}\right) / E_{2}\right]^{-1}$, where $E_{1}$ and $E_{2}$ are the Young's moduli, and $\nu_{1}$ and $\nu_{2}$ are the Poisson ratios of the sphere and plane, respectively. Finally, $z_{0}$ represents the equilibrium spacing for the interaction potential of the surfaces. If $\mu>5$, the JKR theory should be valid while, for $\mu<0.1$, the DMT theory should describe the relation between $A$ and $L[20,21]$. As discussed by Greenwood [21], it is difficult to calculate the area of contact in the intermediate case. Greenwood obtained a numerical solution using a Lennard-Jones potential and defined the contact edge as the point of maximum adhesive stress. Greenwood's solution closely resembles Johnson's MD model. In both cases, the variation of $A$ with $L$ appears very close to the shape of the JKR curve for values of $\mu>0.5$. However, the JKR equation does not correctly predict the actual contact area unless $\mu>5$. Therefore, while a measurement of $A$ vs $L$ may resemble a JKR curve, quantitative analysis would be uncertain. This ambiguity is absent if one is firmly in the DMT limit. In this case, $A$ varies with load in a simple fashion: $A=\pi\left(R^{2 / 3} / K^{2 / 3}\right)(L+2 \pi \gamma R)^{2 / 3}$, where $K=\frac{4}{3} E^{*}$. The pulloff force or critical load $L_{c}$ is given by $L_{c}=-2 \pi \gamma R$, obtained by measuring the force in approach-retract displacements of the tip and sample.

The nanometer range of the contact radius in AFM experiments means that the conductance is limited by the contact and not by the bulk conductance of tip or sample. In this limit the contact conductance becomes directly proportional to $A$. However, the proportionality constant is difficult to determine, preventing absolute determination of $A$ with this technique. Nevertheless, at a fixed voltage, the current is always proportional to $A$ for any current transport mechanism [17,22]. Thus, it is possible to equate the variation in current at a constant voltage to the variation in $A$; this relation is used in this paper.

The experiments were performed in an UHV chamber (base pressure $7 \times 10^{-11}$ Torr) equipped with AFM, Auger electron spectroscopy (AES), and low-energy electron diffraction (LEED) [23]. Normal cantilever force constants were taken from the manufacturer and the normal/lateral force ratio was calculated using the method described by Ogletree et al. [24]. Although the absolute accuracy of the forces measured is limited, the relative changes in friction could be accurately determined by using the same cantilever and tip during a series of measurements.

The sample is a type II B diamond(111) single crystal (boron doped), saturated with hydrogen in a plasma $\left(100 \mathrm{~cm}^{3} / \mathrm{min} \mathrm{H}_{2}\right)$ at $800-900{ }^{\circ} \mathrm{C}$. The cleaning procedure and the single-crystal quality are described in detail elsewhere [15]. Silicon cantilevers coated with $\sim 20 \mathrm{~nm}$ tungsten carbide [25] were used in all measurements. The tips were characterized by scanning electron microscopy and AES. Two types of cantilevers were used, with a spring constant of 88 and $0.23 \mathrm{~N} / \mathrm{m}$, respectively. The former cantilever was used for conductance measurements while the latter one was used for friction measurements. Previous UHV-AFM measurements on a Pt(111) sample showed that the tungsten carbide coated tips are conductive and resistant to wear [26]. The tips were cleaned in UHV immediately prior to the measurements by applying short voltage pulses and/or by rubbing them at high loads on the surface. We built a flexible $I-V$ converter that allows measurements spanning the range from $\mathrm{pA}$ to $\mathrm{mA}$. The friction technique is described in detail elsewhere [5].

Figure 1(a) shows a contact mode AFM image of the $\mathrm{H}$-terminated diamond surface that is consistent with previous work [15]. It is seen to consist of flat (within $3 \AA$ ) islands of $\sim 150-250 \AA$ diameter separated by trenches $\sim 6 \AA$ deep and $\sim 20 \AA$ wide. A $(1 \times 1)$ LEED pattern was observed, indicating that the surface consists of regions of well-ordered diamond(111). Figure 1(b) represents the simultaneously recorded friction map. A magnified friction map of a single island is shown in Fig. 2. All friction and local conductance measurements were acquired on such well-defined islands.

Figure 3 shows many $I-V$ curves recorded at different loads up to $1.7 \mu \mathrm{N}$, using an $88 \mathrm{~N} / \mathrm{m}$ cantilever. The $I-V$ characteristics are semiconductorlike, consistent with the $p$-type doping of the sample. We can now plot the load dependence of the current at several voltages applied to the sample, e.g., at $+4,+3.5$, and $+3.0 \mathrm{~V}$, as shown in the inset of Fig. 3. The data can be fitted very well with the DMT model (solid lines in the inset of Fig. 3) when we use $L_{c}$ as a free parameter. The values of $L_{c}$ deduced from the fits are identical to $0.1 \mu \mathrm{N}$ independently measured from the force-distance curves with the same lever. It is significant that the fits at all voltages have the same $L_{c}$, which confirms that current is proportional

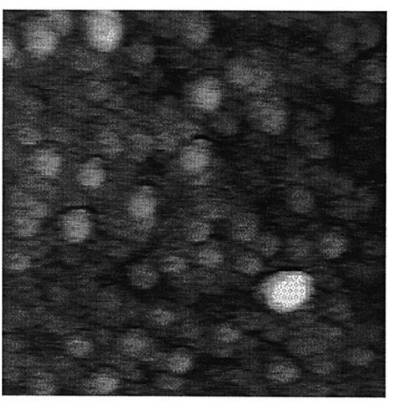

(a)

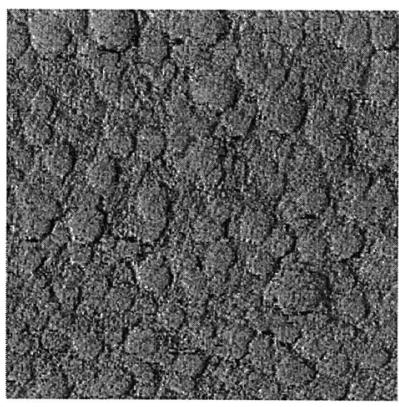

(b)
FIG. 1. (a) Topographical contact mode AFM image of the hydrogen-terminated diamond(111) surface. The surface of the island is flat within $3 \AA$. (b) Simultaneously measured friction map. The surface consists of islands or domains of $\sim 250 \AA$ size. The applied load is $\sim 1 \mathrm{nN}$ and the image size is $2000 \AA \times 2000 \AA$. 


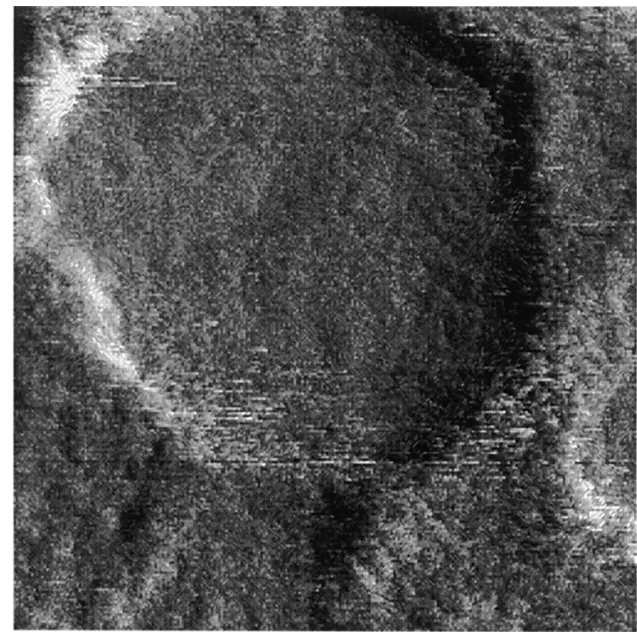

FIG. 2. Friction map of an individual island. All friction and local contact conductance measurements were performed on similar islands. The image size is $300 \AA \times 300 \AA$.

to contact area. These results show unambiguously that the load dependence of the contact area for this single asperity interface can indeed be described by the DMT model. Furthermore, in the inset of Fig. 3, we also fitted the first curve with the JKR model using the constraint $L_{c}=0.1 \mu \mathrm{N}$. The incompatibility of the JKR model applied to our single-asperity contact is clearly shown by the graph. We also found from the fitting statistics that the mean square deviation of the JKR fit is more than 1 order of magnitude higher than that of the DMT. Moreover, a

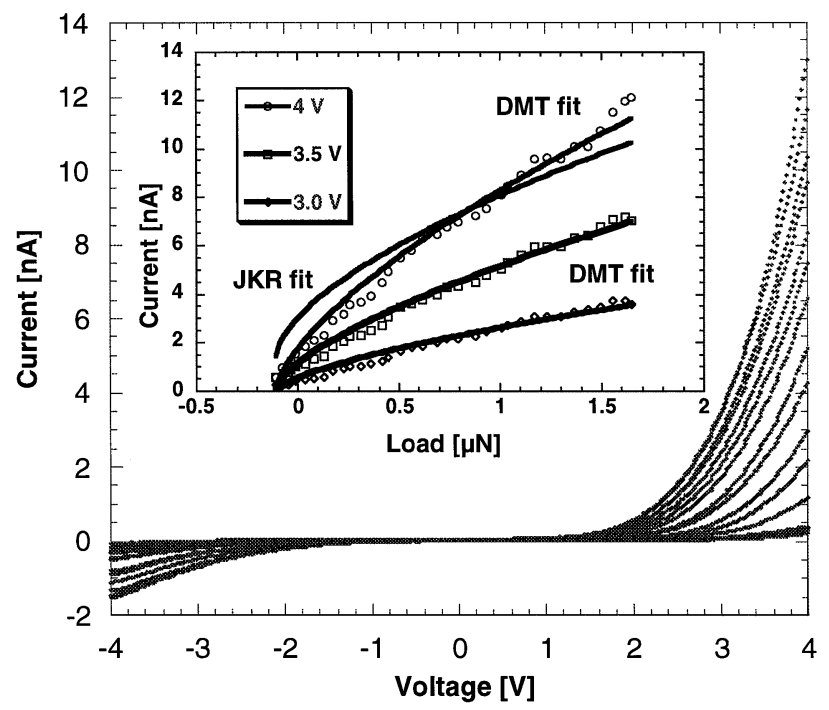

FIG. 3. A family of $128 I-V$ curves recorded as a function of increasing load up to $1.7 \mu \mathrm{N}$, while the sample was biased (for clarity, only each seventh curve is shown). Each curve shows a clear semiconductorlike behavior. The inset represents the current measured through the tip-sample contact vs load, at different constant voltages. Note the accurate fit of the current, which is proportional to the contact area, to the prediction of the DMT continuum mechanics model. A JKR fit, inserted only for the first set of experimental data, is clearly incompatible with this hard, stiff contact.
JKR fit using $L_{c}$ as a free parameter predicts a value quite different from the independently measured $L_{c}$.

Friction measurements were performed using a $0.23 \mathrm{~N} / \mathrm{m}$ cantilever to enhance the sensitivity to frictional forces. The maximum applied load during the friction experiments was $20 \mathrm{nN}$, well within the wearless friction regime. The radius of curvature of the parabolicshaped tip was obtained by scanning over the sharp edges of a faceted $\mathrm{SrTiO}_{3}(305)$ sample [5], and found to be $110 \pm 10 \mathrm{~nm}$. The radius of curvature of the tip was measured before and after tip-sample contact, and no evidence of wear was observed. Using $L_{c}=-2 \pi \gamma R$, we can obtain $\gamma$ from the measured pulloff force from force vs displacement experiments. For $L_{c}=-7.3 \mathrm{nN}$ (inset of Fig. 4) and $R=110 \mathrm{~nm}$, we find that $\gamma=0.01 \mathrm{~J} / \mathrm{m}^{2}$. Using $z_{0}=2 \AA, E_{\text {diamond }}=1164 \mathrm{GPa}, v_{\text {diamond }}=0.08$ [27] and $E_{\text {tip }}=714 \mathrm{GPa}, v_{\text {tip }}=0.24$, we find that $\mu=$ 0.019 . Indeed, this value is much smaller than the DMT condition $\mu<0.1$ discussed above, showing that the contact is firmly in the DMT regime, and that the DMT model applies.

Figure 4 shows the results of frictional force measurements as a function of applied load, which were reproducible at different locations on the sample. The data in Fig. 4 were obtained by decreasing the load from $12 \mathrm{nN}$ to negative loads (unloading). Experiments when the load was increased (loading) exhibited the same behavior as shown in Fig. 4, indicating that the deformation of the contact is elastic for the loads investigated. The data in Fig. 4 can be fitted with the DMT model, treating both $\gamma$ and the shear strength $\tau$ as free parameters, demonstrating that friction is proportional to $A$. The fit (solid line) results in a pulloff force of $-7.3 \mathrm{nN}$ and a shear strength of $238 \mathrm{MPa}$. The contact area is $3.9 \mathrm{~nm}^{2}$ at zero applied load (due to adhesive forces) and $7.4 \mathrm{~nm}^{2}$ at the maximum applied load of $12 \mathrm{nN}$. No friction data for loads

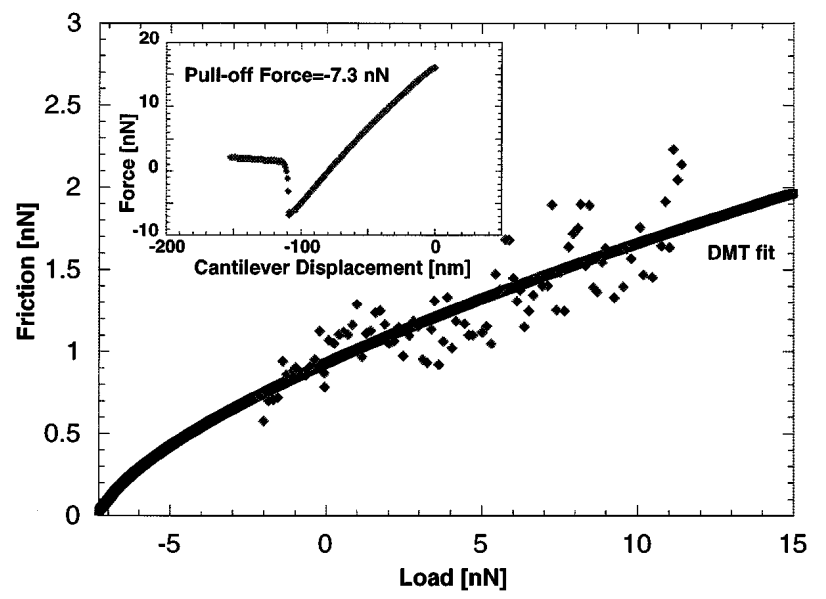

FIG. 4. Friction vs load showing the same dependence as that of contact conductance vs load, and therefore the same dependence predicted by the DMT model, showing that friction is proportional to the contact area. The inset shows a forcedistance curve with the pulloff force, in very good agreement with the value obtained from the DMT fit. 
smaller than $-2 \mathrm{nN}$ could be obtained due to a premature pulloff of the tip. Premature pulloff is promoted by the tip-sample movement during scanning. Force-distance curves involve less lateral movement and therefore provide a direct determination of the pulloff force. The result is shown in the inset of Fig. 4, and shows a pulloff force of $-7.3 \mathrm{nN}$, in excellent agreement with the value obtained from the DMT fit. The measured pulloff force is an independent verification of the DMT fit, since $\gamma$ (and thus also the pulloff force) was treated as a free parameter. Attempts to fit the JKR model to the friction vs load curves, using both $L_{c}$ as a free parameter and as a constrained parameter, produced strongly inconsistent fits.

In conclusion, we have studied the nanotribological behavior of a well-defined hydrogen-terminated diamond(111)/tungsten carbide contact in UHV as a function of applied load. Contact conductance measurements provided a direct and independent way of measuring the area of contact. Current (contact area) versus load curves for a variety of voltages were unambiguously fit by the DMT model, in agreement with the finding that $\mu=0.019<0.1$. The load dependence of the friction was also found to be in excellent agreement with the DMT model, verified by an independent measurement of the pulloff force. Therefore, we conclude that, for this ideal single asperity contact, i.e., one of the hardest, stiffest known heterocontacts involving materials of a great tribological importance, friction is proportional to $A: F_{f}=\tau A$, where $\tau=238 \mathrm{MPa}$ for loads up to $12 \mathrm{nN}$. Our results constitute the first observation in UHV of a load dependence of the contact area described by the DMT continuum mechanics model.

This work was supported by the Director, Office of Energy Research, Office of Basic Energy Sciences, Materials Sciences Division, of the U.S. Department of Energy under Contract No. DE-AC03-76SF00098. The authors thank Dr. P. Reinke for the loan of the diamond sample. R. J. A.v.d.O. acknowledges the support of the Netherlands Organization for Scientific Research (NWO). R.W.C. acknowledges the support of the Natural Sciences and Engineering Research Council of Canada.

*Permanent address: Department of Physics, University of Bucharest, P.O. Box MG-11, Bucharest, Romania.

†Department of Physics, Eindhoven University of Technology, 5600 MB Eindhoven, The Netherlands.

†Present address: Sandia National Laboratories, Mailstop 1413 Albuquerque, NM 87185.

$\S$ To whom correspondence should be addressed. Electronic address: salmeron@stm.lbl.gov

[1] C.M. Mate, G. M. McClelland, R. Erlandsson, and S. Chiang, Phys. Rev. Lett. 59, 1942 (1987).

[2] U.D. Schwarz, O. Zwörner, P. Köster, and R. Wiesendanger, Phys. Rev. B 56, 6997 (1997); U.D. Schwarz,
O. Zwörner, P. Köster, and R. Wiesendanger, Phys. Rev. B 56, 6987 (1997).

[3] M. Binggeli and C. M. Mate, Appl. Phys. Lett. 65, 415 (1994).

[4] M.N. Gardos, in Synthetic Diamond: Emerging CVD Science and Technology, edited by K. E. Spear and J.P. Dismukes, The Electrochemical Society Series (Wiley, New York, 1994).

[5] R.W. Carpick, N. Agraït, D.F. Ogletree, and M. Salmeron, J. Vac. Sci. Technol. B 14, 1289 (1996).

[6] K. L. Johnson, K. Kendall, and A. D. Roberts, Proc. R. Soc. London A 324, 301 (1971).

[7] M. A. Lantz, S. J. O'Shea, M.E. Welland, and K. L. Johnson, Phys. Rev. B 55, 10776 (1997); M. A. Lantz, S. J. O'Shea, and M. E. Welland, Phys. Rev. B 56, 15345 (1997).

[8] D. Maugis, J. Colloid Interface Sci. 150, 243 (1992); K. L. Johnson, Proc. R. Soc. London A 453, 163 (1997).

[9] K. L. Johnson, Contact Mechanics (Cambridge University Press, Cambridge, England, 1987).

[10] B. V. Derjaguin, V.M. Müller, and Y.P. Toporov, J. Colloid Interface Sci. 53, 314 (1975); V. M. Müller, B. V. Derjaguin, and Y.P. Toporov, Colloids Surf. 7, 251 (1983).

[11] M.F. Ashby and D. R. H. Jones, Engineering Materials: An Introduction to Their Properties and Applications (Pergamon Press, Oxford, 1980).

[12] H. Seki, G. M. McClelland, and D. C. Bullock, Wear 116, 381 (1987); H. Tsai and D. B. Bogy, J. Vac. Sci. Technol. A 5, 3287 (1987).

[13] M. M. Schwartz, Ceramic Joining (ASM International, Materials Park, Ohio, 1990).

[14] G. J. Germann, S. R. Cohen, G. Neubauer, G. M. McClelland, H. Seki, and D. Coulman, J. Appl. Phys. 73, 163 (1993).

[15] R. J. A. van den Oetelaar and C. F. J. Flipse, Surf. Sci. 384, L828 (1997).

[16] M.D. Perry and J. A. Harrison, J. Phys. Chem. B 101, 1364 (1997), and references therein.

[17] F. P. Bowden and D. Tabor, The Friction and Lubrication of Solids: Part II, International Series of Monographs on Physics (Oxford University Press, Oxford, 1964).

[18] R. W. Carpick and M. Salmeron, Chem. Rev. 97, 1163 (1997), and references therein.

[19] V.M. Müller, V.S. Yushenko, and B. V. Derjaguin, J. Colloid Interface Sci. 77, 91 (1980).

[20] D. Tabor, J. Colloid Interface Sci. 58, 2 (1977); K. L. Johnson, Langmuir 12, 4510 (1996).

[21] J. A. Greenwood, Proc. R. Soc. London A 453, 1277 (1997).

[22] S. M. Sze, Physics of Semiconductor Devices (Wiley, New York, 1981), 2nd ed.

[23] Q. Dai, R. Vollmer, R. W. Carpick, D. F. Ogletree, and M. Salmeron, Rev. Sci. Instrum. 66, 5266 (1995).

[24] D. F. Ogletree, R. W. Carpick, and M. Salmeron, Rev. Sci. Instrum. 67, 3298 (1996).

[25] NT-MDT Co., Zelenograd Research Institute of Physical Problems, Moscow, Russia.

[26] M. Enachescu, R. W. Carpick, D. F. Ogletree, and M. Salmeron, (to be published).

[27] C. A. Klein, Mater. Res. Bull. 27, 1407 (1992). 